\title{
Análisis funcional de casos de conductas de juego en niños y jóvenes de Bogotá, Colombia
}

\author{
Blanca Patricia Ballesteros, Mónica Ma. Novoa, Claudia Caycedo, Dennys del Rocío García \\ Departamento de Psicología \\ Pontificia Universidad Javeriana de Bogotá
}

Enviar correspondencia a:

blanca.ballesteros@ javeriana.edu.co

\section{RESUMEN}

Se presentan los resultados del análisis funcional de seis casos que participaron en la investigación de mayor escala sobre juegos de suerte, azar y habilidades y suerte con jóvenes de 10 a 22 en la ciudad de Bogotá, Colombia. Los seis casos representan variabilidad en los juegos, excepto el caso 2 de una joven de 17 años, quien solo juega lotería. Los menores de edad participaron con consentimiento informado de los padres, quienes también fueron entrevistados. Se utilizaron entrevistas, una escala de opinión, una escala de situaciones con el juego, observaciones directas y autoregistros de ocasiones de juego durante un periodo de una semana a diez días. El análisis funcional, como metodología para explicar la conducta de personas o grupos, permitió comprender las variables micro y macro contextuales que en cada caso mantienen relaciones funcionales con la conducta de juego y apuesta; además permitió establecer que la mayoría de los casos corresponden a la categoría de juego problema en riesgo de juego patológico. Algunos resultados son consistentes con los reportados por otros autores desde marcos conceptuales distintos, mientras otros señalan particularidades importantes de tener en cuenta al momento de hacer conclusiones y proponer alternativas para intervenciones y futuras investigaciones. Este estudio es pionero en Colombia y en varios lugares por su metodología desde el marco conceptual del Análisis del Comportamiento.

Palabras-clave: Análisis funcional, conducta de juego, contingencias de refuerzo, práctica cultural.

\section{ABSTRACT}

Results are presented from the functional analysis of six individual cases who participated in the larger scale research on games of chance and gambling by young people between 10 and 22 years, their skills and luck in Bogotá, Colombia. The six cases represent gaming variability, except case 2, a 17-year-old female who only plays lotteries. Minors participated with the informed consent of their parents, who were also interviewed. We used interviews, an opinion scale, a gaming situations scale, direct observations and self-recording of gambling frequencies over a period of eight to ten days. Functional analysis was used as a methodology to explain human and group behavior and to understand the micro and macro contextual variables functionally related to gaming and gambling behaviour in each case; it also enabled most of the subjects to be classified in the category of problematic gambling with a risk of pathological gambling. Some results are consistent with those reported by other authors from different conceptual frameworks while others indicate particularities which should be taken into consideration when reaching conclusions and proposing alternatives for intervention and future research. This study is a pioneer of its kind, in Colombia and in several places, for its methodology within the conceptual framework of behaviour analysis.

Keywords: Functional analysis, gambling behaviour, reinforcement contingencies, cultural practice.
E proyecto de investigación de mayor escala del cual se deriva este artículo obedeció a la necesidad de iniciar en Colombia, comenzando en Bogotá, un análisis cuidadoso de las prácticas de juego en las tres categorías explicitadas en la legislación Colombiana: juegos de suerte y azar, de destreza (videojuegos) y de suerte y habilidad (máquinas), asumiendo la definición de juego del Decreto 0114 del 9 de marzo de 1988: "Todo mecanismo o acción basado en las diferentes combinaciones de cálculo y de causalidad, que de lugar a ejercicio recreativo donde se gane o se pierda, ejecutado con el fin de entretenerse, divertirse y/o ganar dinero o especie", teniendo en cuenta los datos de otros países y las demandas de atención psicológica relacionadas con las conductas de juego.

En general en la literatura especializada hay acuerdo sobre la función recreativa y socializadora del juego, pero también se reconoce la susceptibilidad del 
ser humano a volverse dependiente o adicto a condiciones especiales que adquieren valor de refuerzo o de estimulación. Independientemente de las posibles explicaciones a partir de la teoría, es un hecho que la apertura de casinos, salones de bingo, centros de máquinas tragamonedas o tragaperras y otros, así como el crecimiento de la industria de los videojuegos, ha llevado a un cambio en la forma de percibir y valorar las prácticas de juego en la sociedad.

Relacionado con lo anterior, es importante anotar que varias organizaciones en numerosos países han llamado la atención sobre el incremento de conductas de juego que pueden clasificarse como problema, no solo individual, sino comunitario, debido a sus implicaciones y su relación con otros problemas, como otras adicciones, delincuencia, ausentismo escolar y laboral, problemas familiares, ansiedad, depresión y suicidio (Anderson y Bushman, 2001; Bushman y Anderson, 2002; Casado, 2001; Echeburúa, 2000: Ibáñez y cols. 2001; Myers, 2002; Rodríguez Guarín y Gómez Restrepo, 1998; Secades y Villa, 1998; Sherry, 2001; Slater, 2003; Villoria López, 1998). En el estudio español sobre videojuegos, coordinado por Elena Rodríguez (2002), 43.9\% de 3000 jóvenes entre 14 y 18 años reportó haber tenido algún problema por causa de su dedicación al juego y se concluye que los problemas están relacionados con un clima en el cual resulta normal cierto nivel de actitudes violentas, machistas y discriminatorias, es decir, un clima de permisividad, basado en percibir los juegos como sólo diversión, "no real". El problema de la diferencia entre fantasía y realidad, o juego y realidad también es analizado por Goldstein (2001), para quien la gente es capaz de distinguir la violencia simbólica o fantástica implicada en los videojuegos y la violencia real observada en las noticias y en documentales. Para este autor en los juegos electrónicos no hay una víctima viviente ni la intención de dañarla o matarla, componentes indispensables de la definición de conducta violenta en la psicología; en conclusión, no podría hablarse de matar o de violencia en el sentido literal de la palabra. Para Goldstein el problema ha sido confundir entre juego agresivo y conducta agresiva y dice que la evidencia en los estudios en los cuales se explicita esta diferencia señala que los juegos con contenido violento efectivamente inciden en el juego agresivo, pero no afectan la conducta agresiva. Sobre este punto la controversia continúa pues hacen falta estudios longitudinales a partir de esta generación. Los estudios de caso contribuyen a dilucidar este aspecto.

Respecto de los videojuegos, la literatura especializada en la década de 1980 y primeros años de 1990 no es consistente sobre la relación entre la dedicación de los niños y jóvenes a esta práctica de juego y los problemas psicológicos, además de advertir acerca de la carencia de evidencia sobre relaciones causales (Arbinaga, 1996; Estallo, 1994; Funk, 1992; Mcloure y
Mears, 1984, 1986; Provenzo, 1991). Sin embargo, en los últimos años parece haber reportes sobre relaciones más significativas y advertencias más contundentes a padres de familia sobre la vigilancia necesaria de posibles efectos de los videojuegos en el comportamiento de sus hijos/as, como lo anotan Anderson y Dill (2000) y Anderson y Bushman (2001); no obstante, las conclusiones no son del todo consistentes, como se observa en los meta-análisis de los autores anteriores y el de Sherry (2001) (ver también Vessey y Lee, 2000; Cantor, 2003).

Aunque existen algunas controversias respecto de los criterios de diagnóstico de los problemas de juego, parece haber acuerdo con asumir estos problemas en la categoría de adicciones, especialmente porque cumplen con los criterios de tolerancia y síndrome de abstinencia. No obstante, autores como Blaszczynski y Nower (2002), Rodríguez (2002) y varios conductistas (Hayes, Strosahl y wilson, 1999; Ribes Iñesta, 1990) coinciden más en enfoques multidimensionales que en enfoques categóricos o taxonómicos, propios de la tradición del modelo médico. También deben tenerse en cuenta los instrumentos de evaluación utilizados, como lo advierte Becoña (2004), entre otros autores.

Sobre las variables emocionales, algunos estudios se han centrado en las relaciones entre el juego de apuestas, la impulsividad y las estrategias de afrontamiento (Lightsey y Hulsey, 2002), la irritabilidad y la búsqueda de sensaciones (Slater, 2004). Según Potenza (2002), pocos estudios se han dirigido a estudiar el papel que cumplen características como la frecuencia, disponibilidad, preferencias, tipo de juego y dimensiones contextuales que parecen estar muy relacionadas con el desarrollo, el curso y mantenimiento de los problemas de juego. Por otra parte, Lightsey y Hulsey (2002) parten de considerar que los modelos conductuales dejan por fuera las variables cognoscitivas y otras del ambiente, distintas de las contingencias de reforzamiento, sin embargo, no referencian autores desde el interconductismo ni desde las perspectivas contextualistas actuales, de manera que mantienen la tradicional confusión entre los modelos conductuales como si todos hicieran la dicotomía entre conducta y cognición, al llamar conducta solamente a los actos motores observables por terceros y como si las contingencias de reforzamiento solamente se limitaran a relaciones de contigüidad temporal.

En este estudio se asume que el comportamiento está en función de diversas variables que mantienen cierto tipo de relaciones entre ellas (relaciones funcionales), por lo tanto, para explicarlo se requiere identificar dichas relaciones funcionales, lo que a su vez implica comprender las variables o factores no como eventos aislados, sino como eventos con funciones específicas, razón por la cual el método es el análisis 
funcional/contingencial (Balcázar, Suárez-Balcázar y Keys, 1998; Ballesteros, Contreras, Vargas, Palacios \& Bonilla, 2002; Biglan, 1995; Fawcett, 1994; Ribes Iñesta, 1990).

Como actividad humana, el juego es una clase funcional de conducta susceptible de este tipo de análisis y dada su importancia clínica y social, resulta primordial estudiarlo con miras a proveer alternativas de intervención con el fin de prevenir los diversos problemas que cada día muestran una mayor prevalencia, especialmente entre los jóvenes.

Respecto de los factores contextuales, la situación paradójica de la alta fuente de ingresos estatales provenientes de los juegos de azar es referenciada por varios autores sobre el tema pues aparece como una característica común a varios países, incluido Colombia, lo cual lleva a poner atención a las actividades de monitoreo, vigilancia y formación que serían necesarias para abordar esta situación paradójica (por ej. Stinchfield, 2002). Esto implica una competencia de metacontingencias, definidas como la conjunción de contingencias con resultados grupales, de forma que implica conexiones entre prácticas culturales y sus resultados agregados, de lo cual se deriva su importancia en la comprensión de prácticas culturales, entre ellas el juego, y los efectos de retroalimentación, de forma que los resultados agregados inciden en las prácticas culturales (ver Ballesteros, López y Novoa, 2003; Glenn, 1988).

En Colombia se desconocen las características de la situación de juego en la población y su impacto en la salud pública, por lo cual la investigación realizada constituye una de las primeras aproximaciones al problema y es pionera en la perspectiva del análisis del comportamiento y las prácticas culturales, con una metodología multi-fuente y multi-método, en un diseño mixto con niveles de análisis descriptivo, correlacional y funcional, con 217 jóvenes varones y 80 mujeres entre 10 y 22 años de edad, divididos en tres grupos de edad.

El objetivo de este artículo es presentar el análisis funcional de seis casos individuales derivados de la investigación de mayor escala ya referenciada, con el fin de analizar las conductas de juego en las categorías de suerte y azar, habilidades y destrezas y suerte, en su dimensión funcional, en relación con variables micro y macro contextuales.

\section{MÉTODO}

Dentro de la metodología de investigación científica se clasifica el análisis funcional como estudio de caso único, en esta oportunidad, de seis casos indi- viduales. El análisis funcional permite comprender la conducta de individuos o grupos en términos de las contingencias que dieron lugar a ella y explican su mantenimiento. (Ballesteros de Valderrama, 2002; Hayes, Barlow y Nelson-Gray, 1999)

\section{Participantes}

Seis jóvenes residentes en la ciudad de Bogotá, entre 13 y 22 años de edad, incluida una mujer de 17 años. La participación fue voluntaria mediante firma de consentimiento informado. Los menores de edad contaron con consentimiento de sus padres, quienes también participaron en la investigación. Los participantes se seleccionaron intencionalmente de la muestra de la investigación referenciada, con base en los resultados iniciales de la evaluación, con el criterio de frecuencia de juego, variabilidad de juego, disposición para realizar los registros y representación de los grupos de edad (de 10 a 13; 14 a 17 y 18 a 22 años).

\section{Instrumentos}

- Entrevista semiestructurada para los jóvenes y los padres. Esta entrevista incluyó apartados para preguntas sobre variables sociodemográficas, familiares, consumo de sustancias, aspectos escolares/laborales, variables psicológicas y prácticas de juego.

- Entrevista clínica.

- Formatos de registro de observaciones directas en los sitios de juego, diseñados especialmente para evaluar las características topográficas y funcionales en contexto.

- Formatos de autoregistros donde los participantes describieron las condiciones situacionales de juego, sus conductas (hacer, sentir, pensar) durante el juego y las consecuencias.

- Escala de opiniones sobre el juego, para mayores de 14 años y padres. Fue adaptada del Cuestionario sobre hábitos del estudio de Navarra (Urdániz, Izco, Muñoz y Salinas, 1997), con autorización de los autores. Cuenta con 14 ítems de los cuales 7 son de dirección positiva y 7 de dirección negativa. Se obtuvo un índice de confiabilidad con Alfa de Cronbach de 0.72 (Durán, García, Rivera, Waldrón, Ballesteros y Cortés, 2004)

- Escala de Situaciones con el Juego que cuenta con 13 ítems de dirección positiva, adaptada de la utilizada por Moore y Ohtsuka (1999). Es una escala de medición unidimensional sobre el grado de implicación en diversas situaciones relacionadas con el juego y grado de reconocimiento de problemas de control de tales situaciones. Su índice de confiabilidad con Alfa de Cronbach fue de 0.86 (Durán, García, Rivera, Waldrón, Ballesteros y Cortés, 2004). 


\section{Procedimiento}

Una vez terminada la evaluación general de los 297 participantes de la investigación referenciada, con base en los criterios descritos anteriormente se llamó telefónicamente a 10 de los participantes para indagar sobre su interés en continuar con el estudio. De estos se presentan seis de los ocho que completaron toda la evaluación. Las entrevistas y observaciones fueron realizadas por psicólogos con entrenamiento por parte de los investigadores principales. Estas actividades de evaluación se realizaron en el contexto natural de cada participante (residencia, lugares de juego).

\section{RESULTADOS}

Se presentan los análisis funcionales para cada caso con los datos relevantes y el resumen de la información de las entrevistas y registros en los diagramas, con base en las categorías utilizadas por Mattaini y Thyer (1996) y Mattaini (1996) que permiten visualizar los factores implicados en cada caso en sus dimensiones micro y macro y así comprender las prácticas de juego para cada caso. Los diagramas agrupan los factores individuales (historia, condiciones de motivación y ocasiones de refuerzo, reglas y consecuencias) y contextuales (factores estructurales) que en cada caso están relacionados con la conducta de juego. En la descripción de los casos no se incluye información resumida en las gráficas, con el fin de evitar repeticiones.

\section{Caso 1}

Hombre de 22 años, estudiante universitario de ingeniería de sistemas, sin responsabilidad económica en su familia. Vive con su padres y hermanos, es el intermedio de 3 hijos. Dice compartir con su familia como primera actividad los fines de semana y con sus amigos entre semana.

Las razones que dio para jugar se relacionaron con entretenerse, hacer amigos y las emociones que siente; la mitad de las veces lo hace para conseguir dinero y otras veces porque no puede parar. Otros comportamientos asociados son el consumo de bebidas alcohólicas varias veces a la semana y fumar 5 cigarrillos diarios; refiere haber fumado marihuana alguna vez.

Después de la observación de un episodio de juego de una hora y diez minutos, reporta sentir "la adrenalina por todo el cuerpo y las manos heladas, sin sudor". Dice, "Es que fuera de que soy hiperactivo, como que me pongo más, yo no sé, todo raro, como ansioso se debe llamar eso".

Describe su primera ida al casino como muy excitante, por características del contexto como ruidos y luces. En esa ocasión asistió con su primo y ganó mucho dinero: "Como nos fue tan bien, lo primero al salir fue pensar a qué hora íbamos a volver". Desde entonces ha tenido temporadas de juego intenso y también períodos críticos "tocaba amarrarse las manos para no caer en la tentación". Habla de una época durante la cual jugó bastante y perdió dinero, dice que tuvo muchos problemas y se veía obligado a decir muchas mentiras.

Reconoce que "se le va mucha plata". Lo que gana vuelve a jugarlo o lo gasta en pagar las deudas o en rumba. Su mejor amigo también "se envició". En una ocasión se golpearon y rasgaron la ropa para simular que habían sido atracados y explicar la pérdida de un dinero del padre del amigo. Informa haberse sentido muy culpable y mal por esto.

Sobre su vida afectiva y social, se resalta que hace un año tiene novia. Tiene pocas amistades cercanas. Su mejor amigo ya no vive en la ciudad, aunque mantiene contacto frecuente. Con él y su primo es con quienes ha tenido las experiencias más intensas de juego.

Este caso se ubica en el perfil superior de juego, según los criterios establecidos en la investigación de mayor escala: jugar por dinero en uno o más tipos de juego, historia de juego de más de 4 años, frecuencia de juego superior a dos horas semanales y 4 horas en fin de semana, adicionalmente juegan por socialización, emociones y tienen características de impulsividad. Este caso cumple criterios descritos en la categoría de juego patológico (APA, 2000).

\section{Caso 2}

Mujer de 17 años, estudiante de 10 grado, estrato socioeconómico medio. Vive con su madre y dos hermanos, padre fallecido hace tres años.

Inicia juegos de perinola, en el colegio, apostando el dinero para la merienda. De ahí pasa a participar "en cuanta rifa había"; en ocasiones rifaba objetos que le regalaban o dinero "por que cuando se rifa plata, la gente se inscribe más. Después me di cuenta que rara vez ganaba, y que era más la plata que me gastaba".

Después de la muerte del padre empezó a apostar más. Las condiciones familiares cambiaron porque él era quien sostenía económicamente la familia. El hermano mayor tuvo que buscar un trabajo, pero dice "lo que le pagan no sirve". La mamá intentó trabajar pero fue muy difícil pues nunca antes ha trabajado.

Su madre desconoce la forma como su hija apuesta y no estaría de acuerdo con sus apuestas. Las juzga como un gastadero de plata que no vale la pena.

Este caso también se ubica en el perfil superior de nuestra clasificación y corresponde con la categoría de juego patológico (APA, 2000). 


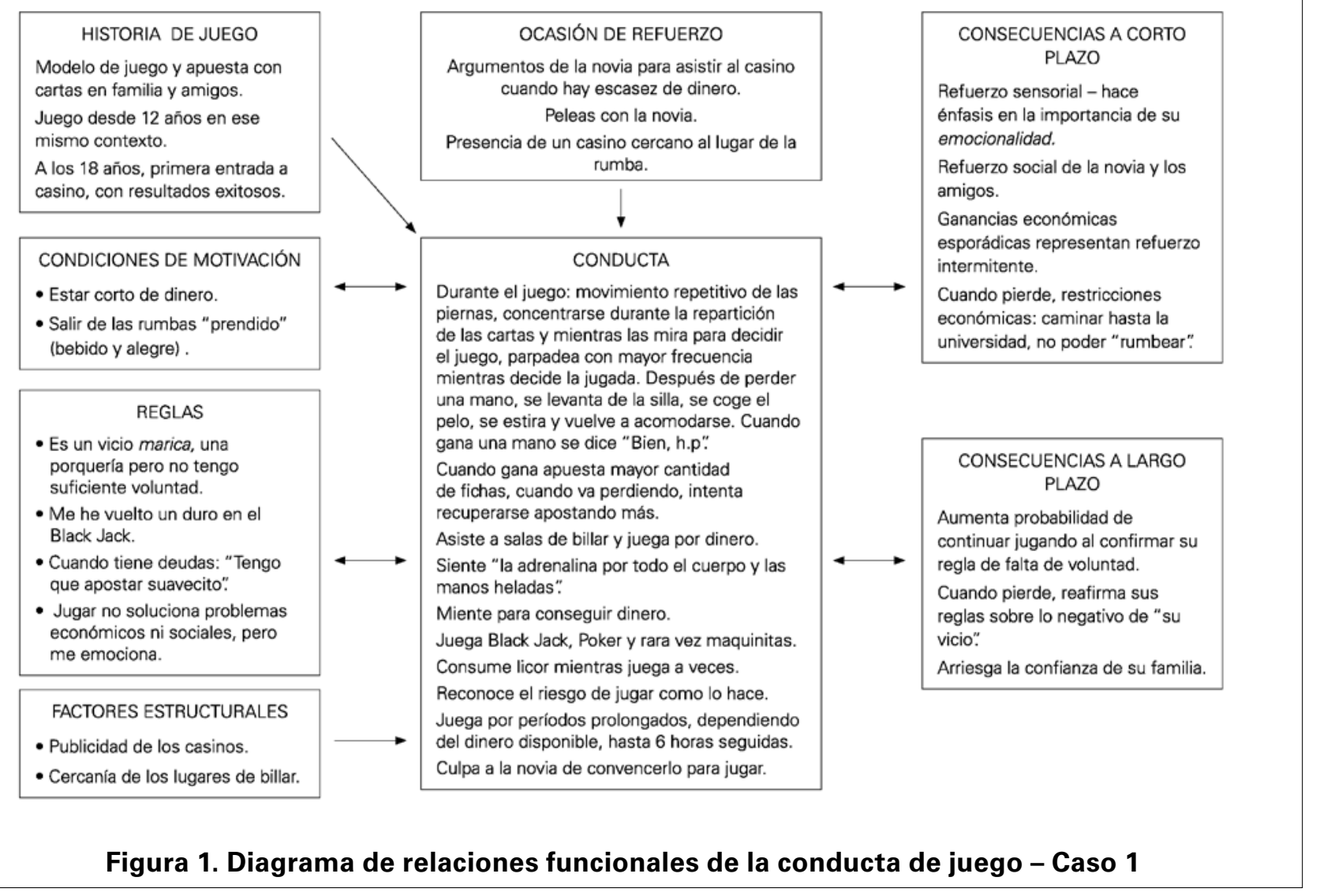

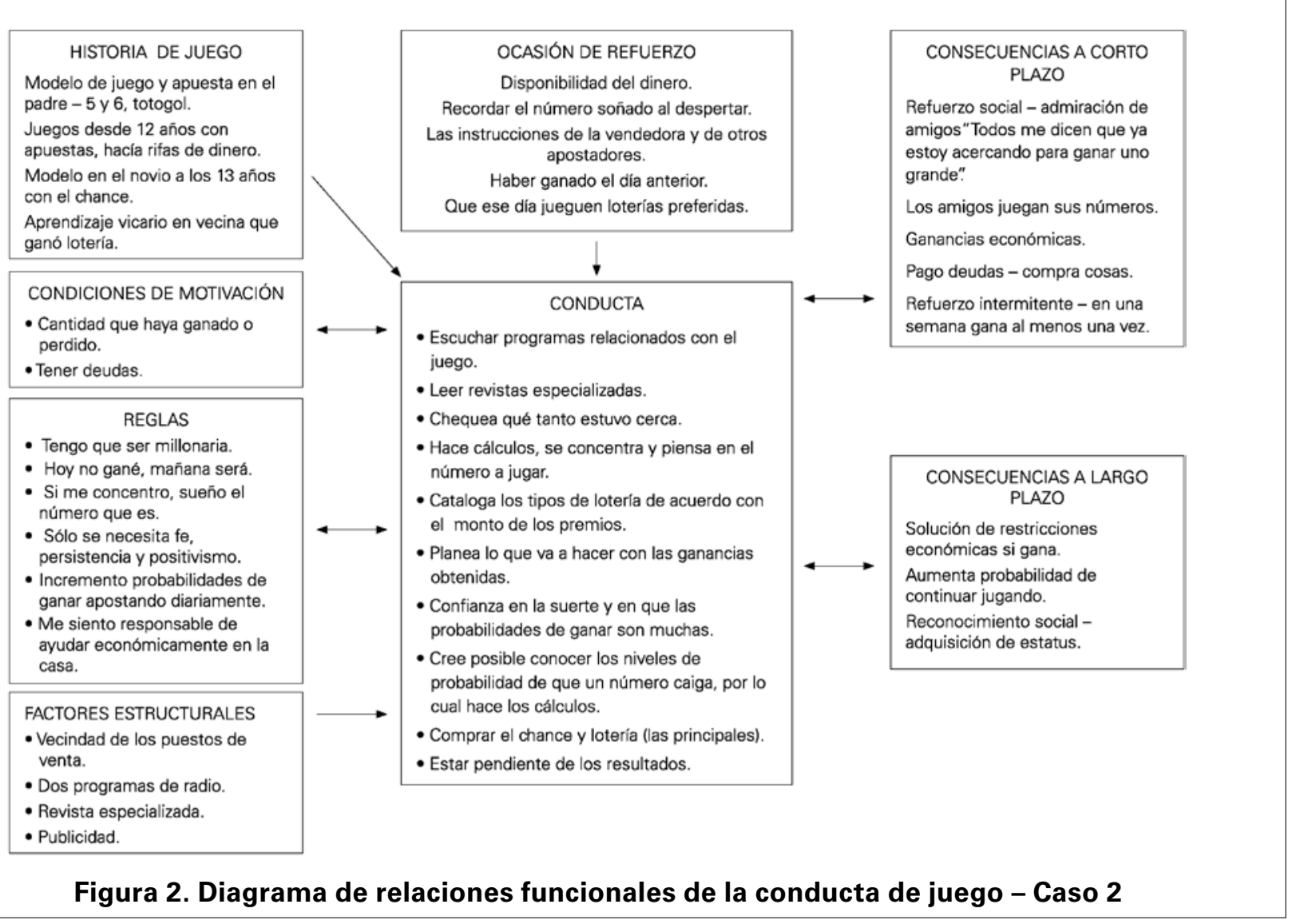




\section{Caso 3}

Joven de 16 años, estudiante de secundaria, en un colegio mixto público; vive con su padre (comerciante), su madre (dedicada al hogar) y su hermano de 10 años. Pertenece al estrato socioeconómico mediomedio.

Hace aproximadamente 1 año empezó a jugar con una frecuencia de 3 a 5 días en la semana, 2 horas entre semana y entre 1 y 2 horas los fines de semana, actualmente ha disminuido la frecuencia de juego a 2 días. Prefiere los videojuegos de carros (carreras o persecuciones) y también practica fútbol. Reporta jugar billar una vez a la semana, con sus amigos a la salida del colegio. Tanto en billar como en fútbol apuesta "la gaseosa" pero nunca dinero.

En cuanto a la relación con su familia, existe buena comunicación y confianza con la madre, mientras la relación con el padre es lejana; aunque comparten algunas actividades y hablan de diferentes temas, no hay confianza sino temor pues lo considera de mal genio. Dice tener pocos amigos, en el barrio sólo comparte con uno de su misma edad, en el colegio habla de un amigo y una amiga cercanos y de un grupo del curso (2 ó 3) con quienes juega, en especial billar. Re- porta tener buenas relaciones con sus compañeros y con sus profesores.

Según el reporte de la madre, su asistencia y disciplina en el colegio han sido buenas y el rendimiento académico aceptable; éste año a raíz de disminución en el rendimiento los padres le restringieron el juego hasta que mejorara; a partir de esto ha mejorado así que se le permitió volver a jugar; ha mantenido un nivel académico aceptable pues se ha recuperado en varias materias. La madre ve el juego como una forma que tiene su hijo de entretenerse, pero sabe que debe ejercer control sobre esta actividad.

Este caso corresponde a la tipología de jugador social en la categoría de juegos de habilidad, y el riesgo está en la prioridad del juego sin la presencia de otras importantes.

\section{Caso 4}

Joven de 14 años, vive con su padre (profesional desempleado a la fecha de la evaluación), su madre (profesional), sus 2 hermanas mayores y otros familiares; pertenece al estrato socioeconómico medio; se encuentra iniciando secundaria un colegio mixto privado.

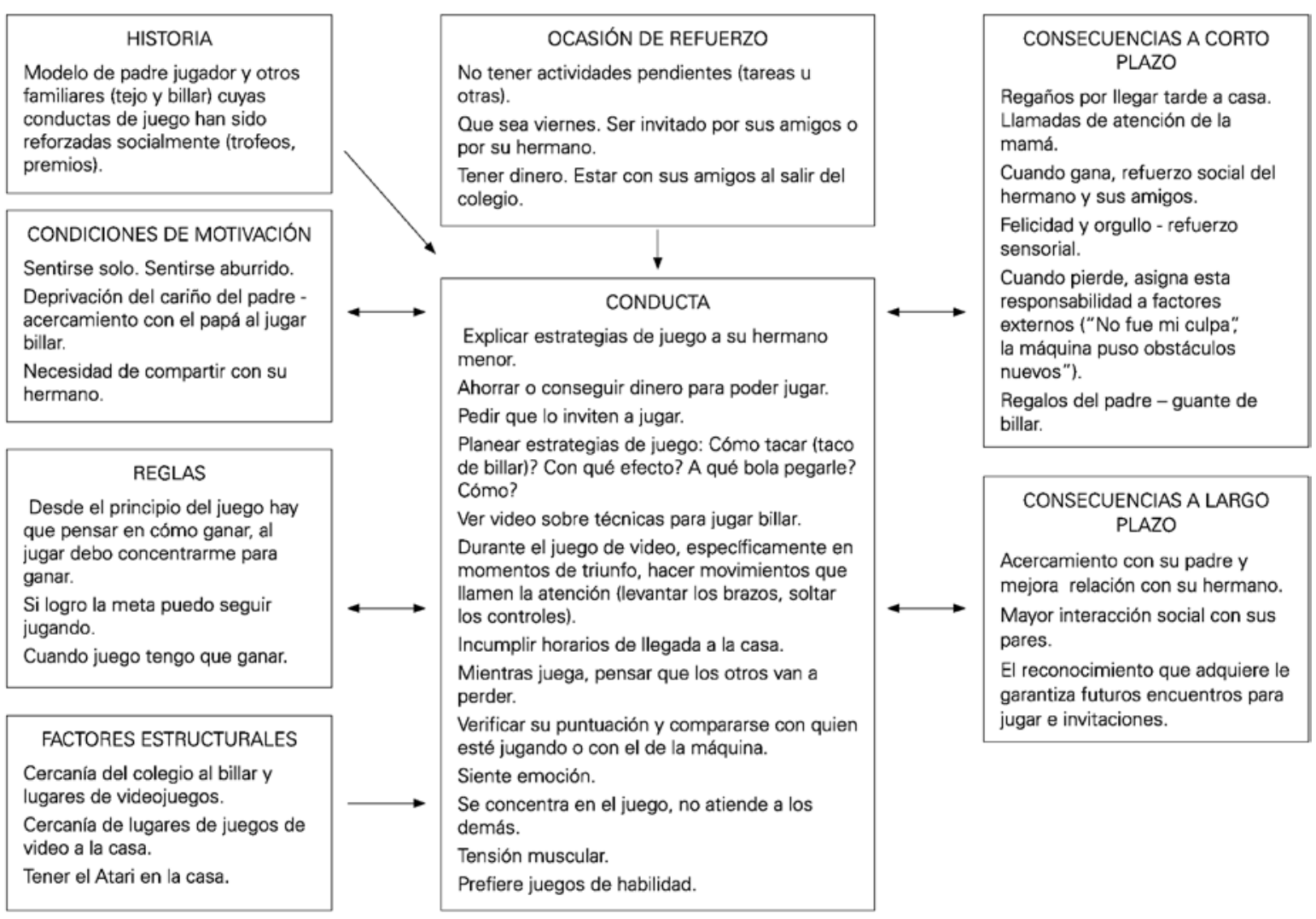

Figura 3. Diagrama de relaciones funcionales de la conducta de juego - Caso 3 


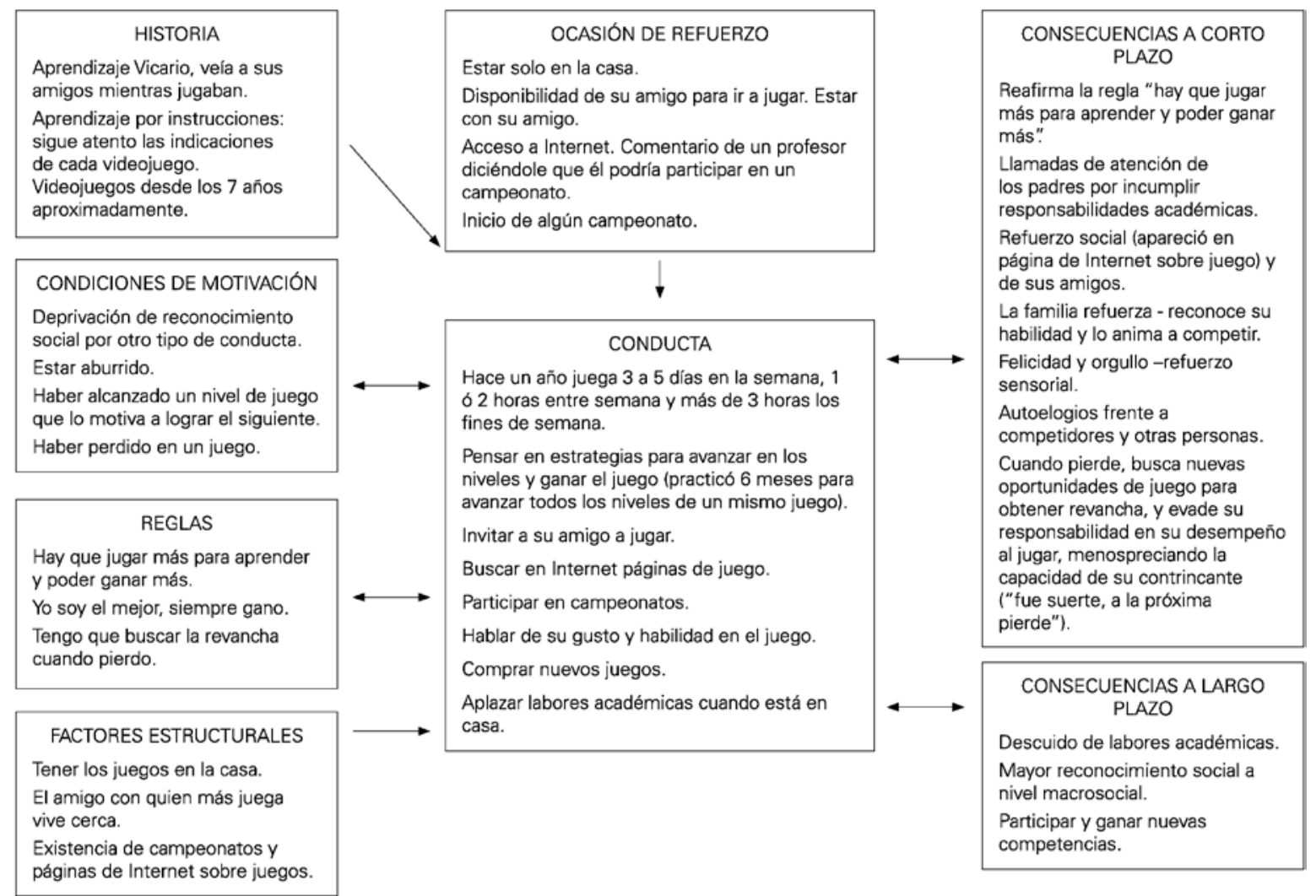

Figura 4. Diagrama de relaciones funcionales de la conducta de juego - Caso 4

En vacaciones juega casi todos lo días hasta 3 horas seguidas y normalmente alrededor de 2 horas. Prefiere los videojuegos de deportes (fútbol) y de estrategia, también practica ping-pong. No suele apostar pero sí invierte dinero en comprar nuevos juegos (hasta $\$ 50.000 .00$ mensuales).

En cuanto a la relación con su familia, existe un estrecho vínculo con la madre, con quien tiene buena comunicación y confianza, él la percibe comprensiva. La relación con el padre es cercana, hay buen trato y confianza; él menciona flexibilidad por parte de su papá, lo que se corrobora con el relato de la madre quien dice que es ella quien lo debe regañar porque el papá casi no lo hace. En relación con las hermanas existe cercanía y a veces comparten actividades como la práctica del ping-pong.

Respecto del área social, menciona pocos amigos, en el barrio comparte el gusto por los videojuegos con uno de 15 años con quien juega casi siempre y generalmente le gana. También menciona a un primo con el que se ve poco. Su relación con compañeros de colegio la califica como buena, aunque no comparte actividades extraclase; con los profesores dice tener una relación lejana, aunque ha hablado con alguno de ellos, quien le contó sobre el campeonato en el que participó. Según el reporte de la madre, su asistencia al colegio es buena pero la disciplina y el rendimiento académico han sido apenas aceptables, ella menciona que después de algunos llamados de atención está cumpliendo más con sus tareas porque a principio de año le dedicaba más tiempo a jugar.

Este caso no puede considerarse en la categoría de juego patológico, pero sí de juego problemático, con alto riesgo de convertirse en patológico debido a la aprobación familiar del juego, a que el juego es la única fuente de reforzamiento social y aplazamiento de actividades académicas.

\section{Caso 5}

Joven de 14 años, menor de dos hijos en una familia monoparental, padres separados. Vive con su madre y hermano mayor. Cursa $8^{\circ}$ grado en un colegio mixto. Lleva una buena relación con su padre con quien se ve semanalmente. De igual forma la relación con su mamá y su hermano son descritas como buenas, basándose en la responsabilidad, el respeto y la comunicación, aunque también reconoce mentir sobre el juego, por historia de restricciones al respecto.

Juega diversos juegos (Yugi-Oh, juegos de play station como Fantasy Tactics, Dance revolución, 


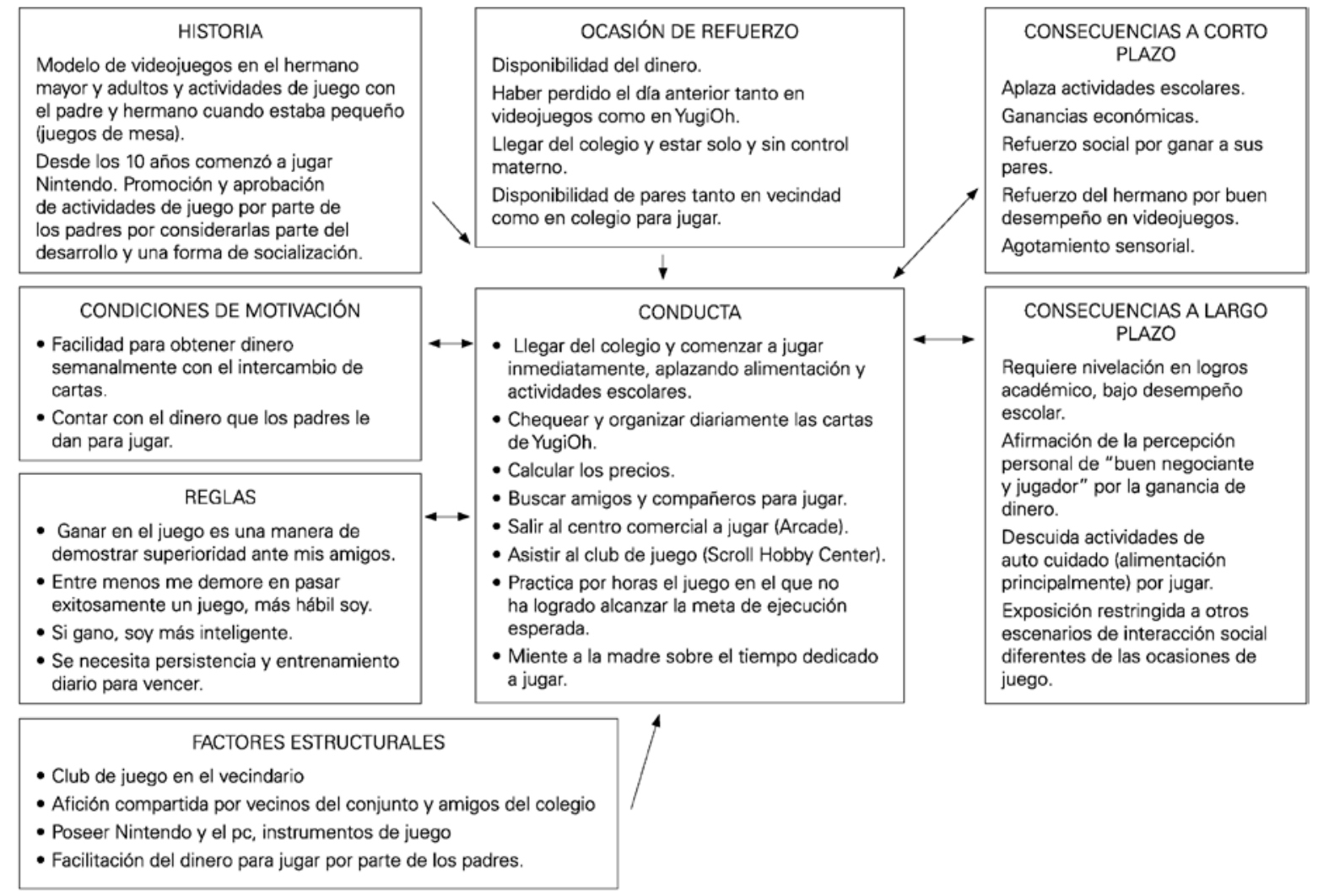

Figura 5. Diagrama de relaciones funcionales de la conducta de juego - Caso 5

Takken 3, Bust a groove, Nascar rumble, Tenchu, Castlevania, megaman x,5 megaman $\times 6$, Metal slug, Yugi.Oh). Por el que más afición muestra es el YugiOh, que tiene cartas de valores elevados que compra, vende e intercambia con amigos, compañeros y gente que va a Scroll (club de juego). Ha llegado a hacer negocios por mas de $\$ 150.000$ en menos de una semana.

Su afición por el juego la comparte con sus compañeros de clase y con su hermano quien también juega bastante e incluso le ha enseñado algunos juegos. No acostumbra apostar en los videojuegos, el dinero que gasta es en maquinitas o en negociar las cartas.

Este caso, por las restricciones respecto de la edad en el sistema de clasificación DSM-IV-RT no puede ser clasificado como jugador patológico, sin embargo, tiene altos indicadores de riesgo, por la cantidad de dinero, la aprobación social, la historia de juego, sus criterios de auto-evaluación y de juicio de los demás y otros descritos en la figura 5 .

\section{Caso 6}

Joven de 13 años, hijo único en familia monoparental. Vive con la madre, padre fallecido hace tres años, separados dos años antes. La madre es permisiva en relación con el juego argumentando que son juegos creativos; la relación es calificada como cariñosa, con buen trato y buena comunicación. El patrón de solución de conflictos reportado en casa se da a través del diálogo y la negociación.

Académicamente el joven se encuentra cursando 8 grado, con bajo rendimiento. Es de menor tamaño que lo esperado para su edad, la relación con sus pares se establece a raíz del juego, tiene contacto interpersonal superficial. Reporta que sus compañeros le dicen que es raro. La madre lo describe como niño inquieto y "respondón" desde pequeño.

Las características de su conducta de juego son indicativos de alto riesgo de llegar a ser jugador patológico, especialmente por los criterios de problemas de impulsividad, la intensidad y frecuencia y la dependencia emocional.

\section{DISCUSIÓN}

El análisis funcional de casos individuales como metodología permitió comprender y explicar la conducta de juego en sus dimensiones formales y fun- 


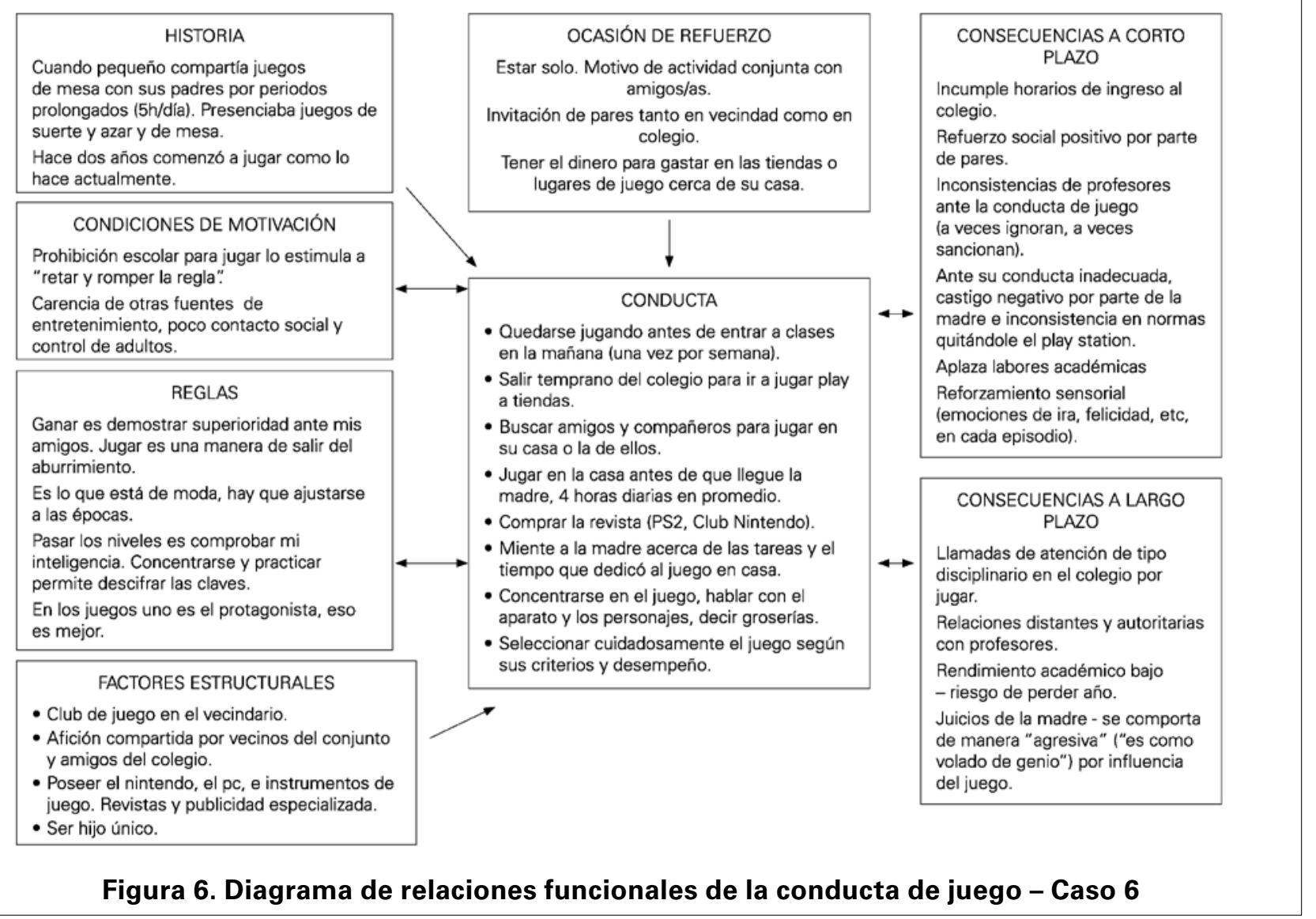

cionales, al identificar de forma precisa los factores históricos y contextuales de los cuales es función esta conducta en cada caso.

Los casos son representativos de las distintas modalidades de juego explicitadas en la legislación Colombiana. Para el caso de juego de suerte y azar se encuentran el 2 (chance y lotería), para los juegos de habilidad y suerte los casos 1 (casino) y los casos 4,5 y 6 (videojuegos) y para los de destreza, el caso 3 (billar). Es importante mencionar que el único caso que tuvo exclusividad en una categoría de juego fue el caso 2. También son representativos de las categorías de juego problemático o de los factores de riesgo descritos en la literatura especializada.

Como se observa en los diagramas de cada caso, aunque formal o topográficamente hay similitudes entre algunos de ellos, cada uno mantiene dimensiones funcionales particulares en la forma como se relacionan las distintas variables. Por ejemplo, para el caso 2, la mujer que juega chance y lotería, el refuerzo sensorial no se constituye en el factor de mantenimiento más importante como si sucede para la mayoría de los otros casos, especialmente para el caso 1 (casino).

En los casos analizados es interesante la comunalidad en términos del reforzamiento sensorial como variable mantenedora de los diversos tipos de prácticas de juego. Por ello, además de analizar las contingencias socialmente mediadas (sea atención social o escapar de una tarea escolar), vale la pena señalar que el reforzamiento automático o sensorial, el cual no está socialmente mediado, se deriva automáticamente de la conducta, razón por la cual para su modificación se requiere implicar, por ejemplo, la alteración de operaciones de establecimiento, sustituyendo refuerzos, aumentando o disminuyendo niveles de estimulación y/o utilizando refuerzo diferencial en cualquier programa de intervención y/o prevención que se plantee sobre el problema. Se postularía que las prácticas de juego en los jóvenes pueden entonces, tener una importante fuente de reforzamiento operante basada en la respuesta bioquímica del organismo, con base en Cataldo y Harris (1982). Por lo tanto, dos funciones posibles de la alta intensidad y duración de la conducta de juego podrían consistir en activar la liberación de endorfinas que pueden producir una sensación "placentera" (reforzamiento positivo) o bien atenuar el dolor (reforzamiento negativo) como sucede en los casos 5 y 6 , donde a pesar de presentarse alteraciones sensoriales, agotamiento, encalambramiento de manos y otro tipo de desgastes y/o lesiones biológicas, los jóvenes continúan practicando por horas 
(Koepp y cols., 1998, ver también Ibañez, Blanco y Sáiz-Ruiz, 2002; Nower, Derevensky y Gupta 2004).

De todas formas, no es suficiente afirmar que la conducta autoestimulativa sea explicada por el desequilibrio de los sistemas neuronales, es imprescindible integrar las características del contexto, especialmente las derivadas de las variables metacontingenciales. De tal manera, se concluye igualmente que en varios de los casos, la prácticas de juego parecen incrementar el nivel de activación/ocupación en un entorno subestimulante, pues el juego, especialmente de la tipología de habilidades (videojuegos) mantiene un nivel de activación que compensa la estimulación ambiental limitada (ver cuadros de ocasiones de juego). Esta hipótesis parece mostrar respaldo en el caso 6 , donde la topografía de la práctica de juego muestra que el entorno poco estimulante produce mayor duración de la misma y aplazamiento de otros comportamientos propios de la edad (labores escolares, alimentación, etc). Se puede afirmar que las prácticas de juego son función de las consecuencias perceptuales interoceptivas y exteroceptivas producidas directamente por el tipo de juego, siendo de naturaleza auditiva, táctil, visual y/o vestibular, como sucede en juegos de habilidades sensorio motoras. El aspecto de la estimulación sensorial también ha sido tratado respecto de los video juegos, por autores como Slater (2003) quien asume la búsqueda de sensación como un factor disposicional de base biológica. Desde el análisis del comportamiento, el concepto de refuerzo sensorial permite explicar todas aquellas conductas clasificadas en esta categorías de búsqueda y de experiencia de sensaciones.

Por otra parte, en todos los casos el reforzamiento social aparece como mantenedor de las prácticas de juego, a veces bajo la forma de atención por parte de los padres, otras veces por parte de los pares o ambos. Para los casos 4, 5 y 6, la práctica de juego se funda en el principio de la diversión, el aprovechamiento del tiempo libre bajo el argumento del aburrimiento y el interés por destacarse en un campo que entre sus pares es de relevancia y proporciona prestigio y una posición privilegiada ante quienes lo practican. Sin embargo, no se ha documentado ningún estudio en el cual se describa la persistencia de un problema de juego en ausencia de consecuencias sociales. De igual forma, la función de las condiciones estructurales en términos de disponibilidad son evidentes en todos los casos.

Respecto de la explicación sobre el mantenimiento de las prácticas de juego en ausencia de reforzamiento en las situaciones en las cuales se espera recompensa, o en las que se programan recompensas de un modo no sistemático (como en el chance y el juego en casino), se hipotetiza que la "frustración" es conducta operante para la cual la retirada del refuerzo es una operación de establecimiento (Michael, 1982, 1993), pues la estimulación aversiva (perder) es la operación de establecimiento típica como condición motivacional.

Con el propósito de integrar los aspectos señalados, se puede sugerir que las conductas de juego evolucionan gracias a varias combinaciones de tipo biológico y ambiental, de manera que podrían platearse tres momentos en el desarrollo de las conductas problemáticas de juego. En un primer momento, durante una etapa menor en el desarrollo cronológico y social, la exposición a múltiples eventos sociales, especialmente de tipo familiar, en los cuales se incorporan las reglas asociadas con la práctica frecuente como un tipo de actividad normal, adaptativa y socialmente deseable. En un segundo momento, los jóvenes exhiben patrones de juego como respuesta a la subestimulación y el juego se comienza a considerar una respuesta compensatoria que en algunos casos puede ser motivo de preocupación para padres o terceros significativos, dado su impacto perturbador en otras actividades propias de la edad, como cumplir tareas académicas y de interacción social. En este momento, la conducta de todas formas ya ha adquirido funciones sociales de tipo macrontingencial, que escapan a las contingencias medioambientales inmediatas administradas por parte de padres y profesores. Finalmente, podría hablarse de un último momento, en el cual el patrón de juego se cronifica y en virtud del reforzamiento intermitente, como el dinero obtenido y el entretenimiento social, se constituye en un problema.

Al respecto, Blaszczynski y Nower (2002) son partidarios de hablar de subtipos de jugadores y proponen tres vías para diferenciarlos, pero especialmente consideran que el modelo de vías proporciona un marco conceptual integrador de la información proveniente de la clínica y de la investigación, el cual queda disponible para ser probado empíricamente y orientaría la acción clínica. El caso 3 y el 2 pueden ser ejemplo de la primera vía. Los casos 4 y 6 se ajustarían a la segunda vía y finalmente para la tercera vía un ejemplo se ve en el caso 5. Respecto de estos subtipos, es necesario desarrollar estudios en los cuales se comparen los diversos tipos descritos, permitan corroborar el modelo y derivar las alternativas de intervención.

La literatura especializada en el tema del juego ha reportado como factores comunes a los problemas de juego los antecedentes de juego en la familia como predisponente importante (Hardoon, Gupta y Derevensky, 2004, Nower, Derevensky y Gupta, 2004), lo cual es consistente con lo encontrado en los casos analizados, donde existió exposición y modelamiento de padres principalmente en juegos de mesa y juegos de apuestas. Esto es relevante al pensar en progra- 
mas de prevención, en la dirección que mencionan Winters, Stinchfield, Botzet y Anderson (2002).

Otro aspecto de interés en la literatura especializada es la conducta de consumo de sustancias asociada con los problemas de juego. De los casos analizados, solamente el caso 1, tal vez por ser el único caso mayor de edad, presenta consumo de alcohol y cigarrillo asociado con sus prácticas de apuestas en casino y billar. El análisis de este caso permite considerar lo que autores como Vitaro, Ferland, Jacques y Ladouceur (1998) y Nower, Derevensky y Gupta (2004) han reportado respecto del consumo de sustancias psicoactivas y el problema de control de impulsos.

Sobre la relación frecuente en la literatura especializada entre problemas de juego y apuestas y ambiente familiar desfavorable, en este estudio no se encuentra un ambiente familiar conflictivo, pero si vale la pena mencionar la característica de estructura uniparental en tres de los casos, especialmente de los menores, la falta de presencia de adultos en la casa durante gran parte del tiempo y el desconocimiento de la magnitud de la conducta de juego y apuesta. En este sentido, varios estudios y pronunciamientos disponibles han hecho ver la importancia de monitorear el curso de las conductas de juego, pues aunque algunos apostadores en vez de mostrar progreso en sus apuestas, disminuyen su grado de apuestas, en otros casos el curso es progresivo, con el riesgo de conductas socialmente inaceptables. En el caso 1 puede verse este aspecto con claridad, en el evento narrado sobre engaño y mentiras para explicar perdida de dinero.

Respecto del llamado a estudiar mejor la relación entre los factores de riesgo y los problemas de juego, este estudio permite entender en cada caso la forma como los denominados factores de riesgo se relacionan funcionalmente para comprender las particularidades de la conducta como proceso integral, y también permite incluir en el análisis los factores estructurales facilitadores de la ocurrencia de los episodios de juego. Como se mencionó anteriormente, y lo referencian autores como Najavits, Grymala y George (2003), Rodríguez (2002) y Winters, Stinchfield, Botzet y Anderson (2002), cada vez parece haber mas aceptación social de las distintas modalidades de juego. Desde la perspectiva de este trabajo, lo anterior hace que se instauran como prácticas culturales mantenidas por metacontingencias poderosas como las políticas económicas y la legislación en el campo de la salud pública, incluida la recreación. De esta forma, la persona está expuesta a una variedad de condiciones estimulares que facilitan la conducta, la cual se hace altamente probable cuando no existen repertorios de auto control. Es decir, cuando las condiciones estimulares en el contexto confluyen con condiciones de motivación, con historia de juego y con ocasiones de refuerzo, hablamos de una situación que involucra conducta de elección y decisión.

Sobre el tema de las creencias irracionales de control asociadas con problemas de apuestas en jóvenes (Moore y Ohtsuka, 1999), en el caso 2 se evidencian, por ejemplo, en la creencia en la solución de los problemas económicos mediante las apuestas. Sin embargo, vale la pena aclarar que las creencias se formulan desde nuestra perspectiva conductual como reglas que guían la conducta. Esta ilusión de control es una conducta gobernada por reglas, con características de conducta supersticiosa, resistente a la extinción por estar bajo contingencias de refuerzo social y económico importantes (en programa intermitente) y en algunos casos, más que otros, como se mencionó anteriormente, también por refuerzo sensorial (ver Kerr y Keenan, 1997 sobre conducta gobernada por reglas y Rachlin, 2000, sobre autocontrol).

En los casos se diferencian claramente las diversas reglas (creencias) en función de las cuales se mantienen las prácticas de juego, incluyan o no apuestas, pero indudablemente estas reglas a su vez se explican en función de las contingencias de refuerzo vigentes y de la historia de aprendizaje. La regla o creencia por sí sola no tiene poder de explicación causal. De ahí la fortaleza de una explicación en términos de relaciones funcionales.

Sobre las características adictivas de los videojuegos, el análisis de casos permite concluir una congruencia con Renom (2000) cuando menciona el grado de interactividad y el efecto de la motivación inicial, la cual puede ser mejorar la autoestima apareciendo ante los demás como expertos, el aburrimiento o un reto personal. Los resultados de este estudio también son consistentes con Griffiths (1991), Fisher (1994), Griffiths y Minton (1997), Griffiths y Hunt (1998), Ivory (2002) y Derevensky, Gupta y Winters (2003) sobre las características adictivas o de dependencia de los videojuegos y sus semejanzas con las de las apuestas.

Respecto de la función socializadora de los videojuegos violentos, en algunos de los análisis de caso se evidencia lo mencionado por Goldstein (2001), sobre la preferencia de estos juegos en niños y jóvenes, relacionada con la identidad masculina. Los juegos se vuelven tema de conversación y ocasión para integrar grupos de amistad, como se encontró en los casos individuales y en el grupo de participantes de la investigación realizada (Ballesteros, Novoa, Caycedo y García, 2004). Desde la perspectiva asumida en este estudio, es importante atender a las condiciones más duraderas o permanentes en las vidas de los niños y jóvenes, las cuales con mayor probabilidad tendrán efectos sobre la formación de patrones de conducta, incluidas las normas, reglas y valores. Es decir, la atención debe centrarse en todas las con- 
diciones del proceso de socialización como proceso complejo, extendido en el tiempo (ver Roche, BarnesHolmes, Barnes-Holmes y Hayes, 2001). En este sentido, como puede concluirse de estos casos, si jugar juegos violentos no ocupa un espacio importante en la vida de los niños, niñas y jóvenes y el resto de sus actividades pertenece a un contexto con ejemplares claros de conducta social en el amplio sentido de la palabra, los riesgos de problemas disminuyen.

Finalmente, una de las funciones del juego en todas las especies es practicar conductas que se requerirán en la vida adulta e infortunadamente los asuntos de la guerra han sido importantes para la humanidad, de manera que si actualmente se habla de una degradación en el sentido moral, se tendría que analizar con mayor rigurosidad la cantidad de tiempo dedicado a los videojuego, especialmente cuando su contenido es violento y cuando el contexto familiar y social también lo es, como sucede en un país en guerra y con altos índices de violencia familiar, escolar y sociopolítica como Colombia.

Este artículo se deriva de un estudio de mayor escala "Análisis de las Prácticas de Juegos de Suerte y Azar, de Destreza y de Suerte y Habilidad en niños/ as y jóvenes de Bogotá", Código 1203-04-12643, con cofinanciación de COLCIENCIAS, realizado entre enero de 2002 y diciembre de 2004.

\section{REFERENCIAS}

Anderson, C. A. y Dill, K. E. (2000). Video games and aggressive thoughts, feelings, and behavior in the laboratory and in life. Journal of Personality and Social Psychology, 78, 2, $772-790$.

Anderson, C. A. y Bushman, B. J. (2001). Effects of violent video games on aggressive behavior, aggressive cognition, aggressive affect, physiological arousal, and prosocial behavior: A meta-analytic review of the scientific literature. Psychological Science, 12, 353359.

American Psychiatric Association, APA (2000) Diagnostic and statistical manual of mental disorders, DSM-IV-RT. Washington, D. C.: APA.

Arbinaga, F. (1996). El juego patológico en estudiantes menores de 18 años: Incidencia, uso de drogas y variables asociadas. Adicciones, 8, 331-347.

Balcázar, F. E., Suárez-Balcázar, Y. \& Keys, C.B. (1998). Un modelo de investigación-acción para desarrollar la capacidad de comunidades para incrementar su poder. Suma Psicológica, 5, 123-147.
Ballesteros de Valderrama, B. P. (2002). Análisis funcional de un caso clínico. Cuadernos de Clínica \# 7. Bogotá: Pontificia Universidad Javeriana, Javergraf.

Ballesteros de Valderrama, B. P., Contreras, C.E., Vargas, F.J., Palacios, S.R. \& Bonilla, L.P. (2002). La pandilla juvenil: breve revisión y análisis funcional de un caso. Revista Internacional de Psicología Clínica y de La Salud, 2, 2, $335-350$.

Ballesteros de Valderrama, B. P, López L., W. \& Novoa G., M. M. (2003). El análisis del comportamientos en los temas sociales: una propuesta para una cultura de paz. Revista Latinoamericana de Psicología, 35, 3, 299316.

Ballesteros, B. P., Novoa, M., Caycedo, C. García, D. del R. (2004). Análisis de las Prácticas de Juegos de Suerte y Azar, de Destreza y de Suerte y Habilidad en niños/as y jóvenes de Bogotá. Bogotá, Facultad de Psicología, Pontificia Universidad Javeriana, Informe presentado a COLCIENCIAS. Documento inédito.

Becoña, E. (2004). Prevalencia del juego patológico en Galicia mediante el nods. ¿Descenso de la prevalencia o mejor evaluación del trastorno? Adicciones, 16, 3, 173 $-184$.

Biglan, A. (1995). Changing cultural practices. Reno, NV: Context Press.

Blaszczynski, A. y Nower, L. (2002). A pathways model of problem and pathological gambling. Addiction, 97, 487 $-499$.

Bushman, B. J. \& Anderson, C. A. (2002). Violent video games and hostile expectations: a test of the general aggression model. Personality and social psychology bulletin, 28, 12, $1679-1686$.

Cantor, J. (2003). Media violence and its effects on aggression: assessing the scientific evidence. Journalism and Mass Communication Quarterly, 80, 2, $468-470$.

Casado, S. (2001). Qué es la ludopatía? www. juegopatoló gico.com/secciones.

Cataldo, M. F. y Harris, J. (1982) The biological basis for self-injury. Analysis and intervention in developmental disabilities, 2, 21-39.

Derevensky, J. L., Gupta, R. y Winters, K. (2003). Prevalence Rates of Youth Gambling Problems: Are the Current Rates Inflated? Journal of Gambling Studies, 19, 4, 405.

Durán, L., García, A. M. Rivera, C. S. Waldrón, A. M., Ballesteros, B. P. y Cortés, O. F. (2004). Validación de instrumentos para evaluar prácticas de juego en niños, niñas y jóvenes de Bogotá. Trabajo de grado para obtener el título de Psicólogo, Facultad de psicología de la Pontificia Universidad Javeriana de Bogotá. Documento inédito.

Echeburúa, E. (2000) ¿Adicciones...sin drogas? Las nuevas adicciones ( $2^{\mathrm{a}}$ edic). Bilbao: Desclee de Brouwer. 
Estallo, J.A. (1994). Videojuegos, personalidad y conducta. Psichotema, 6, 2, 181-190.

Fawcett, S. B. (1991). Some values guiding community research and action. Journal of Applied Behavior Analysis, 24, 621- 936.

Fisher, S. (1994). Identifying video game addiction in children and adolescents. Addictive Behaviors, 19, 545-553.

Funk, J.B. (1992). Video games: Benign or malignant? Developmental and Behavioral Pediatrics, 13, 53-54.

Glenn, S. (1988). Contingencies and metacontingencies. The Behavior Analyst, 11, 161-179.

Goldstein, J. (2001). Does playing violent video games cause aggressive behavior?. Disponible en http:// culturalpolicy.uchicago.edu/conf2001/papers/ goldstein.html

Griffiths, M. D. (1991). Amusement machine playing in childhood and adolescence: a comparative analysis of video games and fruit machines. Journal of Adolescence, 14, 53-63.

Griffiths, M. D. \& Minton, C. (1997). Arcade gambling: a research note. Psychological Reports, 80, 413-414.

Griffiths, M. D. \& Hunt, N. (1998). Dependence of computer games by adolescents. Psychological Reports, 82, 475-476.

Hardoon, K. K., Gupta, R. y Derevensky, J. L. (2004). Psychosocial variables associated with adolescent gambling. Psychology of addictive behaviors, 18, 2, 170-179.

Hayes, S. C., Barlow, D. H. y Nelson-Gray, R. O. (1999). The scientist practitioner: research and accountability in the age of managed care ( $2^{\text {nd }}$ Edition). Boston: Allyn and Bacon.

Hayes, S.C., Strosahl, K.D. \& Wilson, K.G. (1999). Acceptance and commitment therapy: an experiential approach to behavior change. New York: Guilford Press.

Ibañez, A., Blanco, C., Donahue, E., Lesieur, H.R., Pérez de Castro, I., Fernández, J. \& Sáiz-Ruiz, J. (2001). Psychiatric comorbidity in pathological gamblers seeking treatment. American Journal of Psychiatry, $158,1733-1735$.

Ibañez, A., Blanco, C. y Sáiz-Ruiz, J. (2002). Neurobiology of pathological gambling. Psychiatric Annals, 32, 181 $-185$

Ivory, J. D.(2002). Addictive, but not for me: the thirdperson effect and electronic game players' views toward the medium's potential for dependency and addiction. Disponible en página electrónica unc.edu/ jivory/3rd.html

Kerr, K. P. \& Keenan, M. (1997). Rules and rule-governance: New directions in the theoretical and experimental analysis of human behaviour. En K. Dillenburger, M. F. O'Reilly y M. Keenan (Eds.), Advances in Behaviour
Analysis (pp. 205 - 226). Dublin: University College Dublin Press.

Koepp, M. J., R. N. Gunn, A. D. Lawrence, V. J. Cunningham, A. Dagher, T. Jones, D. J. Brooks, C. J. Bench, \& P. M. Grasby. (1998). Evidence for striatal dopamine release during a video game. Nature, 393, 266-268.

Lightsey, O. R. Jr. \& Hulsey, C.D. (2002). Impulsivity, coping, stress and problem gambling among university students. Journal of Counseling Psychology, 49, 2, $203-211$.

Mcloure, R. F. \& Mears, F. G. (1984). Videogame Players: Personality Characteristics and Demographic Variables. Psychological Reports, 55, 271-276.

Mcloure, R. F. \& Mears, F, G. (1986). Videogame Playing and Psychopatology. Psychological Reports, 59, 5962.

Mattaini, M. A. y Thyer B. A. (1996) (eds) Finding solutions to social problems. Washington: American Psychological Association.

Mattaini, M.A. (1996). Envisioning cultural practices. The Behavior Analyst, 19, 257 - 271.

Michael, J. (1982) Distinguishing between discriminative and motivational functions of stimuli. Journal of the experimental analysis of behaviour, 37, 149-155.

Michael, J. (1993) Establishing operations. The behaviour analyst, 27, 345-355.

Moore, S. y Ohtsuka, K. (1999). Beliefs About Control Over Gambling Among Young People, and Their Relation to Problem Gambling. Psychology of Addictive Behaviors, 13, 4, 339 - 347.

Myers, J. (2002). Royally flushed over youth gambling. The Education Digest, 67, 5, 56 - 61.

Najavits, L. M., Grymala, L. D. \& George, B. (2003). Can advertising increase awareness of problem grambling? A statewide survey of impact. Psychology of addictive behaviors, 17, 4, 324-327.

Nower, L., Derevensky, J. L. \& Gupta, R. (2004) The Relationship of Impulsivity, Sensation Seeking, Coping, and Substance Use in Youth Gamblers. Psychology of Addictive Behaviors, 18, 1, 49-55.

Potenza, M. N. (2002). A perspective on future directions in the prevention, treatment and research of pathological gambling. Psychiatric Annals, 32, 3, 203 -207.

Provenzo, E. (1991). Video Kids. Making sense of Nintendo. Cambridge: Harvard University Press.

Rachlin, H. (2000). The science of self.control. Cambridge: Harvard University Press.

Renom, J. A. (2000). Nuevas patologías en pediatria social: Ciberpatología pediátrica. Ponencia en el XXIXCongreso nacional Ordinario de Pediatría de la AEP. Tenerife, 1417 de Junio. Disponible en la pagina electrónica (www) comtf.es/pediatria/Congreso_AEP_2000/Ponenciashtm/Arguemi_Renom.htm 
Ribes-Iñesta, E. (1990). Psicología y salud: Un análisis conceptual. Madrid: Martínez Roca.

Roche, B., Barnes-Holmes, D., Barnes-Holmes, Y. \& Hayes, S. C. (2001). Social processes. En S. C. Hayes, Barnes-Holmes, D. \& B. Roche (Edt), Relational Frame Theory: A post-Skinnerian account of human language and cognition. (pg. 197- 209). Plenum Press.

Rodríguez Guarín, P. y Gómez Restrepo, C. (1998). Juego patológico: comorbilidad con dependencia de sustancias, trastorno afectivo bipolar y trastorno obsesivocompulsivo. Revista Colombiana de Psiquiatría, 27, 3, $181-196$.

Rodríguez, E. (2002). Jóvenes y videojuegos: espacio, significación y conflictos. España: Instituto de la juventud y Fundación de ayuda contra la drogadicción. Disponible en la página de mtas.es/injuve/biblio/estudio_jnjuve/estudiospdf

Secades, R. y Villa, A. (1998). El juego patológico. Prevención, evaluación y tratamiento en la adolescencia. Madrid: Editorial Pirámide.

Sherry, J. L. (2001). The effects of violent video games on aggression: a meta-analysis. Human Communication Research, 27, 3, 409 - 431.
Slater, M. D. (2003). Alienation, aggression and sensation seeking as predictors of adolescent use of violent film, computer and website content. Journal of Communication, 51, 1, 105 - 122.

Stinchfield, R. (2002). Youth gambling: how big a problem? Psychiatric Annals, 32, 197- 202.

Urdániz, G., Izco, A., Muñoz, O. \& Salinas, M. (1997). Cuestionario sobre hábitos. Estudio de la cultura del juego en Navarra. Pamplona: Fundación Bartolomé De Carranza. Recuperado el 8 de junio de 2003, de http:// www.ctv.es/USERS/ f.carranza/INFO-DEF.doc.

Vessey, J. A. \& Lee, J. E. (2000). Violent video games affecting our children. Pediatric Nursing, 26, 6, 607 611.

Villoria López, C. (1998). Ansiedad y depresión en el juego patológico. Revista electrónica de psicología, V. 2, \#1. En www.psicologia.com

Vitaro, F., Ferland, F., Jacques, C. \& Ladouceur, R. (1998). Gambling, substance use, and impulsivity during adolescence. Psychology of Addictive Behaviors, 12, 3, 185-194.

Winters, K. C., Stinchfield, R. D., Botzet, A. \& Anderson, N. (2002). A prospective study of youth gambling behavior. Psychology of addictive behaviors, 16, 1, 3-9. 